\title{
Neuromonitoring with Intermittent Transcranial Doppler Sonography and Continuous Near Infrared Spectroscopy in Aortic Arch Surgery: An Observational Study
}

Marcus Thudium ( $\square$ marcus.thudium@ukbonn.de)

Universitatsklinikum Bonn https://orcid.org/0000-0002-9881-4875

Evgeniya Kornilov

Weizmann Institute of Science

Tobias Hilbert

Universitatsklinikum Bonn

Georg Daniel Duerr

Universitatsklinikum Bonn

Christopher Gestrich

Universitatsklinikum Bonn

\section{Research article}

Keywords: Aortic dissection, neuromonitoring, transcranial doppler, NIRS

Posted Date: April 22nd, 2020

DOI: https://doi.org/10.21203/rs.3.rs-22203/v1

License: (c) (1) This work is licensed under a Creative Commons Attribution 4.0 International License.

Read Full License 


\section{Abstract \\ Background}

Aortic Arch repair for aortic dissection is still associated with a high mortality rate. Adequate means of neuromonitoring to guide cerebral hemodynamics, especially during selective anterior cerebral perfusion (SACP) are desirable. It is possible that this cannot be achieved by one monitoring modality alone. It was the objective of this study to investigate an easy multimodal monitoring setup consisting of EEG, near infrared spectroscopy (NIRS) and transcranial doppler sonography.

\section{Methods}

Patients with surgery for aortic dissection were included. In addition to standard hemodynamic monitoring, patients received continuous bilateral NIRS, processed EEG with bispectral index (BIS) and intermittent transcranial doppler sonography of the medial cerebral artery (MCA) with a standard B-mode ultrasound device. Doppler measurements were taken bilaterally before cardiopulmonary bypass (CPB), during CPB and during the selective brain perfusion phase in regular intervals

\section{Results}

Six patients with aortic dissection could be included. Two patients died, one in the operating room and one on ICU. Four patients survived without neurological deficit. Two, however, suffered from transient postoperative delirium. Multimodal monitoring led to change in CPB flow or cannula repositioning in 3 patients (50\%). Left sided mean flow velocities of the MCA significantly decreased during selective brain perfusion $(p=0.028)$, as did BIS values $(p=0.028)$

\section{Conclusions}

Multimodal monitoring consisting of BIS, NIRS and B-Mode transcranial sonography can have an impact on hemodynamic management in aortic arch operations. More research is needed to establish target parameters of cerebral perfusion.

\section{Background}

Aortic arch repair for Stanford type A dissection is one of the most invasive procedures currently performed in operating rooms. The procedure requires phases of complete circulatory arrest and selective anterior cerebral perfusion (SACP) via the carotid arteries. The disease is still associated with high mortality rates although these could be reduced in the last years.(1) Especially intraoperative cerebral malperfusion and severe brain injury are one of the most feared complications and mortality causes. For maximum patient safety, an optimal neuroprotection and neuromonitoring protocol would be desirable. 
However, during selective brain perfusion (SBP), only few monitoring options remain, one of them being near infrared spectroscopy (NIRS) and the other transcranial doppler sonography (TCD). While NIRS has become the standard of care in aortic arch repair, this is not the case for TCD. One of the causes for the latter is probably the necessity of having a complex setup for continuous measurements. Other groups have hypothesized that a multimodal approach may be beneficial and could provide more information to guide cerebral perfusion. $(2,3)$ We therefore report our easy monitoring setup feasible in many clinical settings using continuous NIRS and intermittent TCD in patients with aortic arch repair for aortic dissection.

\section{Methods}

The study was approved by the ethics committee of the University of Bonn. Informed consent was waived due to the non-invasive and observational nature of the study and because patients required immediate surgery. Before anesthesia induction, patients received arterial cannulation for continuous blood pressure measurement and standard monitoring according to the American society of anesthesiologists (ASA). Induction was performed with Etomidate and Sufentanil. Patients were orotracheally intubated and received central venous catheterization as well as a urinary catheter.

In all patients the arterial cannula for the cardiopulmonary bypass (CPB) was inserted directly into the right subclavian artery according to the standard protocol. Access to the subclavian artery was achieved by a small incision caudal of the right clavicle.

All patients received median sternotomy and Heparin $400 \mathrm{mg} / \mathrm{kg}$ were injected. The venous two-staged cannula was inserted via the right Atrium. CPB was established, all patients cooled to $25-28{ }^{\circ} \mathrm{C}$ and cross-clamping of the Aorta was performed. Acid-base management was performed using the alpha-stat method. To inspect and repair the aortic arch a short period of complete circulatory arrest was accepted, the brachiocephalic artery was then clamped and selective cerebral perfusion was started at $10 \mathrm{ml} / \mathrm{kg} / \mathrm{min}$. In all cases a second balloon-tipped cannula was inserted into the left carotid artery to insure sufficient blood flow to both hemispheres. For neuroprotection, patients received $500 \mathrm{mg}$ Thiopental and $500 \mathrm{mg}$ Prednisolon.

Prior to skin incision, NIRS optodes were attached bilaterally to the patient's forehead and NIRS was measured continuously. Next to the NIRS optodes, a bispectral index (BIS) electrode was attached and BIS was also measured continuously (BIS Vista, Medtronic, Dublin, Ireland).

For TCD measurements, a regular S5-1 transducer on a CX 50 ultrasound machine (Philips Health Systems, Hamburg, Germany) was used. To reach the medial cerebral artery (MCA), the ultrasound transducer was placed on the temporal fossa slightly above the zygomatic arch and the MCA was located with color doppler. MCA velocity (MCAV) was measured with pulsed wave doppler in a depth of 3,5 to $4,5 \mathrm{~cm}$ where there was little or no angulation between the direction of the ultrasound waves and direction of blood flow. TCD measurements were obtained on both sides of the skull after skin incision, after beginning of CPB, and in 10 min intervals during SBP. Mean flow velocities were registered by hand 
and saved in a standard Excel spreadsheet. To reduce inter-rater variations, all measurements were taken by one investigator experienced in TCD.

Statistical analysis was performed using the SPSS software version 23.0 (IBM, Chicago, IL, USA). Due to a small number of cases only nonparametric statistical tests were used to interpret the data. Data is presented as median (interquartile range) or number (\%). Wilcoxon Signed Rank Test was used to compare BIS, NIRS and MCAV measurements before CPB and during SACP. P values $\leq 0.05$ were considered significant.

\section{Results}

Six patients with Stanford type A dissection were included between January 2018 and March 2019.

Patient 1 was operated due to a retrograde dissection months after an endovascular stent graft repair (TEVAR) of a Type B aortic dissection. The patient was cannulated according to standard and an aortic arch repair was performed using a Thoraflex Hybrid Prothesis. In addition, the patient received an aortic valve repair using a $25 \mathrm{~mm}$ Carpentier Edwards Perimount Prosthesis due to severe aortic regurgitation.

During SACP, NIRS measurements showed a right and left sided reduction of $5 \%$ and $2 \%$, respectively, when compared to measurements undertaken after skin incision.

TCD measurements showed a right sided increase in MCAV of $3 \mathrm{~cm} / \mathrm{s}$ while left sided MCAV decreased by $5 \mathrm{~cm} / \mathrm{s}$. We rated this as stable perfusion and did not alter cannula position or CPB settings. The Patient recovered well and showed no signs of neurological disorder.

In patient 2, operative conditions were difficult due to extreme obesity. This patient was also operated on as redo surgey after retrograde dissection shortly after TEVAR due to Typ B aortic dissection. After standard cannulation, repair of the ascending aorta and the aortic arch was performed using a $32 \mathrm{~mm}$ Hemashield Platinum Prosthesis. The supraaortic vessels were reinserted. During beginning of SACP the NIRS and TCD measurements dropped drastically. Therefore cannulation was altered and blood flow over the CPB was increased. This only lead to an insufficient increase in values leaving rightsided NIRS and TCD values decreased by $22 \%$ and $27 \mathrm{~cm} / \mathrm{s}$ and leftside NIRS and TCD values decreased by $7 \%$ and $8 \mathrm{~cm} / \mathrm{s}$, respectively.

After prolonged CPB time, weaning proved unsuccessful because of right ventricular failure and extracorporeal life support had to be established. In the postoperative course, the patient developed sepsis with subsequent multi organ failure and deceased on ICU.

Patient 3 was cannulated according to standard and the aortic arch was repaired using a Thoraflex Hybrid Prothesis with reinsertion of the supraaortic vessels. During SACP, we only saw a slight decrease in left sided NIRS values by $8 \%$ and stable values on the right side. The TCD measurements showed a right and left sided reduction in MCAV by $36 \mathrm{~cm} / \mathrm{s}$ and $29 \mathrm{~cm} / \mathrm{s}$, respectively. We decided to increase the 
blood flow, tolerated higher NIRS values and used TCD for further references. After successful operation the patient recovered well.

Patient 4 was also operated on due to acute aortic dissection. The patient was cannulated according to standard and the aortic arch was repaired using a Thoraflex Hybrid Prothesis with reinsertion of the supraaortic vessels. During SACP, we

saw an increase in right and left sided NIRS values by 6 and 12\%, respectively. In contrary, we saw reduction of left sided MCAV of $18 \mathrm{ml} / \mathrm{s}$ but an increase of $4 \mathrm{ml} / \mathrm{s}$ on the right side. The cannulas were also not corrected. This patient recovered well, but showed a prolonged but mild delirium.

In patient 5 we performed Aortic arch repair using a $28 \mathrm{~mm}$ Hemashield Platinum Prosthesis. Right sided NIRS values were low at the time of skin incision, probably due to impaired blood flow caused by the dissection. After standard cannulation and during SACP right sided NIRS values increased slightly by $11 \%$. Left sided values increased slightly by $9 \%$. The TCD measurement showed no relevant differences in MCAV in the right side but a drop on the left side by $40 \mathrm{~cm} / \mathrm{s}$. Following the surgeon's statement we did not alter blood flow or cannulation. The patient recovered well from surgery but showed a substantial hyperactive delirium probably due to previous i.v. drug use.

In Patient 6, operative conditions proved to be adverse and the distal end of the aortic prothesis could not be attached without re-dissection. Since SACP time was already prolonged, there was consensus that there was no remaining option for this patient, so death in tabula was diagnosed. In this patient NIRS was reduced during SACP by $15 \%$ and $11 \%$ on the right and left side, respectively. Here again TCD measurements seemed to be more sensitive and showed a severe reduction of mean blood flow velocity starting at a low velocity and still dropping by 27 and $12 \mathrm{~cm} / \mathrm{s}$ on the right and left side, respectively. SACP cannulae had to be adjusted repeatedly after compromised flow was seen in TCD.

Baseline characteristics of the mentioned patients are summarized in Table 1. A representative graph and overview of NIRS measurements during surgery is shown in Fig. 1. 
Table 1

Patient baseline characteristics.

\begin{tabular}{|ll|}
\hline parameter & incidence \\
\hline age ,years & $64(54.5-79.5)$ \\
\hline male sex, $\mathrm{n}$ & $3(50)$ \\
\hline BMl & $22(21.3-41.3)$ \\
\hline Body surface area, $\mathrm{m}^{2}$ & $1.88(1.8-2.53)$ \\
\hline delirium, $\mathrm{n}$ & $2(33.3)$ \\
\hline death, $\mathrm{n}$ & $2(33.3)$ \\
\hline bypass time, h & $4,67(3,27-6,23)$ \\
\hline cross-clamp time, $\mathrm{h}$ & $3,02(2,08-3,25)$ \\
\hline Data presented as median (range) or number $(\%)$. \\
\hline
\end{tabular}

When looking at all patients, MCAV in the left MCA significantly decreased during selective brain perfusion as did BIS numbers. MCAV in the right MCA as well as NIRS measurements also declined during CPB but showed no significant differences. See Table 2 for measurement data.

Table 2

BIS, NIRS and MCAV measurements.

\begin{tabular}{|llll|}
\hline parameter & before CPB & during SCAP & p value \\
\hline NIRS right frontal, \% & $72.3(62.5-77.5)$ & $66.8(57.4-76.8)$ & 0.8 \\
\hline NIRS left frontal, \% & $72.9(62.5-84)$ & $67.5(60.5-80.2)$ & 0.8 \\
\hline MCAV right-sided, cm/s & $35.5(27.9-47.5)$ & $25(14.7-35.2)$ & 0.3 \\
\hline MCAV left-sided, cm/s & $35.1(22.6-53.1)$ & $20.2(14.9-32)$ & 0.028 \\
\hline BIS & $38.5(35.8-40)$ & $4.4(1.5-16.5)$ & 0.028 \\
\hline Data presented as median (range). & & \\
\hline NIRS - Near infrared Spectroscopy & & \\
\hline MCAV- Middle Cerebral Artery mean flow Velocity & \\
\hline BIS - Bispectral Index &
\end{tabular}

In three patients (patients 2, 3 and 6), CPB flow was adjusted or carotid cannulae were repositioned after TCD measurements while in the other patients, adequate MCAV values were confirmed with TCD. Low 
MCA blood flow signal intensity on the colour doppler image also proved to be an important indicator for cannula repositioning in patients 2,3 and 6 .

\section{Discussion}

Aortic arch repair is still associated with a relatively high mortality rate. In addition, inadequate cerebral perfusion during SACP can lead to unfavorable neurological outcome. The best parameters to monitor adequate cerebral perfusion are still unclear. NIRS has become widely used because it is non-invasive, easy to use, and provides a robust signal. However, the validity of the method is still disputed. It was shown that, NIRS can indicate a dislocation of the arterial cannula.(4) Since cerebral oxygenation as indicated by NIRS is the result of several factors, Denault et al developed a protocol for improvement in case of desaturation.(5) Joshi et al. even used NIRS to predict limits of cerebral autoregulation.(6) However, it seems that a constant $\mathrm{rSO}_{2}$ may not guarantee adequate perfusion. We could not see major differences in cerebral oxygenation during the procedure, except during deep hypothermic cardiac arrest, as can be seen in Fig. 1. And while MCAV showed significant decreases on the left side we saw no significant differences in NIRS measurements. This is in line with our previous findings in cardiac operations, where we found only little variation in regional oxygen saturation.(7) It can be interpreted that NIRS indicates major hemodynamic changes while more subtle changes may remain unnoticed by the method. For SACP, we therefore suggest NIRS monitoring as a minimum requirement.

TCD has long been used as a method to assess cerebral hemodynamics.(8) But whether MCAV actually reflects cerebral blood flow is subject of controversy, since basal arteries may also undergo caliber changes.(9) Nonetheless, there is mostly a consensus that TCD can detect changes in cerebral blood flow and is therefore still used in many physiological investigations.(10) The feasibility and benefit of TCD for neuro outcome has been presented by Estrera et al. but with a need of a neurosonographer to operate continuous TCD.(11) It has also been used to monitor cerebral blood flow in aortic arch surgery in a continuous setup.(2) It was our aim to develop a protocol which can improve neuromonitoring using widely available equipment. We therefore chose to use a standard B-mode ultrasound machine with TCD presets. Apart from the advantage that existing devices can be used, B-mode and color doppler facilitates localization of the MCA since the vessel can be localized visually and in difficult cases anatomical landmarks inside the skull can be used to guide the transducer angle and position. However, with this method, continuous measurements are hardly possible. In a report by Ghazy et al., the transducer was fixed with a movable arm that held the probe in place.(3) Thus, the authors could visualize cerebral circulation and decide on a unilateral brain perfusion, since the circle of Willis was intact. We intentionally accepted the disadvantage of non-continuous TCD measurements in trade-off for bilateral TCD. Denault has recently proposed a modified NIRS algorithm in which ultrasound imaging plays a role in hemodynamic evaluation and cerebral imaging in case of increased intracranial pressure.(12) We suggest that ultrasound should become even more central in cerebral monitoring to confirm NIRS findings and to evaluate cerebral hemodynamics. 
Our data shows that TCD seems to be more sensitive to altered cerebral perfusion. While NIRS values react more slowly to reduced perfusion reduced MCAV can be measured promptly. Our significant reduction in left sided MCAV during SCAP is probably explained when looking at the cannulation technique. We cannulated the right subclavian artery with the standard $18 \mathrm{~F}$ or $20 \mathrm{~F}$ cannula but inserted a $10 \mathrm{~F}$ (?) Cannula into the left carotid artery. Blood flow is therefore obviously reduced on the left side due to the difference in cannula diameter, since the perfusionist controls overall flow during SACP, but not in the individual cannula. On the other hand anatomical differences in the arterial vessels may also influence or reduce flow and cause substantial hypoperfusion which needs to be prevented.

That NIRS showed no difference on the left side might be due to compensation via the circle of Willis or show that even if flow is reduced cerebral oxygenation might still be sufficient.

Unfortunately, it remains unknown how to determine adequate cerebral blood flow with TCD. Adding to that, we cannot present data which might have hinted towards TCD values that could have been used as landmarks to determine sufficient perfusion. As much of the decision making depends on trends and the baseline MCAV this is a crucial measurement but also difficult to evaluate, since flow in the cerebral arteries may already be compromised by the dissection. We noticed that pre-CPB MCAV was significantly lower than in the following measurements during CPB. This may be a sign of malperfusion before CPB or could conversely represent hyperperfusion in the following measurements, which may occur during CPB and which we described previously.(13) Wang et al. provide some suggestions on sufficient cerebral blood flow, but also report a close correlation between NIRS and TCD measurements, which we cannot confirm in our patients nor did we observe this preciously.(7) Thus, a wider use of TCD and further investigations will be needed to find definite answers on how to measure adequate cerebral blood flow with TCD of the MCA.

TCD measurements led to adjustment of CPB flow or repositioning of the cannulae in two patients where a flow deficit was not indicated by NIRS. This may be an argument for bilateral continuous TCD monitoring. But apart from the lack in availability, a fixation of the probe may also be impractical, since small movements of the head during surgery may impair the doppler signal and may necessitate regular repositioning of the transducer. For continuous measurement, a headframe with doppler-only probes could also be used and may represent ideal monitoring for SACP. However, attaching the headframe and searching the MCA flow signal can be time consuming and requires special training.(11) Despite some disadvantages B-mode TCD should be used as a standard tool to guide SACP, as Ghazy et al. have also suggested.(3)

While BIS monitoring has also been used and has become part of standard monitoring, its utility to assess cerebral perfusion during hypothermia with Thiopental boluses is reduced. Naturally, BIS values during SACP are significantly lower than in other phases of the operation. Therefore, although BIS can be an indicator of inadequate brain perfusion, in SACP this is not the case. However, BIS can conversely be used as an indicator of the suppressive effect of Thiopental and hypothermia. 
Determination of adequate cerebral perfusion is difficult and may not be reflected by one monitoring modality alone. A multimodal monitoring approach has also been suggested by Zanatta et al. with TCD, NIRS and somatosensory evoked potentials (SEPs).(14) SEP measurements are well validated, but the method requires training, may be time consuming and may be considered impractical for Stanford type A dissections.

In a previous study, we reported that in surgery with CPB, a combination of BIS, NIRS and TCD may increase patient safety.(7) We prpopse that this is also the case for patients undergoing SACP. While BIS is of lesser importance in SACP, NIRS can be used to reflect cerebral metabolism and TCD to reflect cerebral hemodynamics. We show that TCD gives us another tool to make sure that the patients brain is well perfused. However, the relevance for outcome has yet to be established.

There are some limitations inherent in this study. We can draw no conclusion about the outcome relevance of the reported method due to the small number of cases. Although monitoring had some impact on management, its effect on outcome yet remains to be shown. Also, by choosing a noncontinuous TCD measurement approach, there is missing information between measurements which was a trade-off for bilateral monitoring with an existing device. We think that this is acceptable since with NIRS there is still one continuous monitoring modality in place which can at least indicate major changes.

In summary we presented an easy and widely available method of monitoring cerebral perfusion with continuous NIRS and intermittent TCD monitoring in patients undergoing aortic arch surgery for Stanford type A dissection even if more substantial research is necessary to learn more about cerebral perfusion monitoring during SACP.

\section{Conclusions}

Our results suggest that TCD with a B-mode ultrasound device is a useful addition to NIRS in patients undergoing aortic arch surgery and can be used to adjust cerebral hemodynamic management during SACP. Therefore, both modalities should be considered the minimum standard of care in these patients.

\section{Abbreviations}

ASA

American Society of Anesthesiologists

BIS

Bispecral Index

CPB

cardiopulmonary bipass

MCA

middle cerebral artery 
MCAV

middle cerebral artery flow velocity

NIRS

near infrared spectroscopy

$\mathrm{rSO}_{2}$

regional cerebral oxygen saturation

TCD

transcranial doppler

TEVAR

thoracic endovascular aortic repair

\section{Declarations}

\section{Ethics approval}

This study was approved by the ethics committee of the University of Bonn (Chair: Prof. K. Racké), Informed consent was waived due to the observational and non-invasive nature of the study.

Consent for Publication

Not applicable

\section{Availability of data and materials}

The datasets used and analysed during the current study are available from the corresponding author on reasonable request.

\section{Competing interests}

The authors declare that they have no competing interests

\section{Funding}

Departmental funding only

\section{Authors' contributions}

MT designed the study, acquired the data, interpreted the data, drafted and revised the manuscript. EK analyzed and interpreted the data, helped draft and revised the manuscript. TH helped in data acquisition and interpretation and revised the manuscript, GDD interpreted the data and revised the manuscript, CG designed the study, helped in data acquisition, analyzed and interpreted the data, drafted and revised the manuscript. All authors approved the submitted version of the manuscript and agreed both to be personally accountable for their contributions and to ensure that questions related to the accuracy or 
integrity of any part of the work, even ones in which the author was not personally involved, are appropriately investigated, resolved, and the resolution documented in the literature.

\section{References}

1. Pape LA, Awais M, Woznicki EM, Suzuki T, Trimarchi S, Evangelista A, et al. Presentation, Diagnosis, and Outcomes of Acute Aortic Dissection. J Am Coll Cardiol. 2015 Jul;66(4):350-8.

2. Wang X, Ji B, Yang B, Liu G, Miao N, Yang J, et al. Real-Time Continuous Neuromonitoring Combines Transcranial Cerebral Doppler with Near-Infrared Spectroscopy Cerebral Oxygen Saturation During Total Aortic Arch Replacement Procedure: A Pilot Study. ASAIO J. 2012 Apr;58(2):122.

3. Ghazy T, Darwisch A, Schmidt T, Fajfrova Z, Zickmüller C, Mashhour A, et al. Transcranial Doppler Sonography for Optimization of Cerebral Perfusion in Aortic Arch Operation. Ann Thorac Surg. 2016 Jan;101(1):e15-6.

4. Murkin JM, Adams SJ, Novick RJ, Quantz M, Bainbridge D, Iglesias I, et al. Monitoring brain oxygen saturation during coronary bypass surgery: a randomized, prospective study. Anesth Analg. 2007 Jan;104(1):51-8.

5. Denault A, Deschamps A, Murkin JM. A proposed algorithm for the intraoperative use of cerebral near-infrared spectroscopy. Semin Cardiothorac Vasc Anesth. 2007 Dec;11(4):274-81.

6. Joshi B, Ono M, Brown C, Brady K, Easley RB, Yenokyan G, et al. Predicting the limits of cerebral autoregulation during cardiopulmonary bypass. Anesth Analg. 2012 Mar;114(3):503-10.

7. Thudium M, Heinze I, Ellerkmann RK, Hilbert T. Cerebral Function and Perfusion during Cardiopulmonary Bypass: A Plea for a Multimodal Monitoring Approach. Heart Surg Forum. 2018 Jan;21(1):E028-35.

8. Aaslid R, Markwalder TM, Nornes H. Noninvasive transcranial Doppler ultrasound recording of flow velocity in basal cerebral arteries. J Neurosurg. 1982 Dec;57(6):769-74.

9. Weyland A, Stephan H, Kazmaier S, Weyland W, Schorn B, Grüne F, et al. Flow velocity measurements as an index of cerebral blood flow. Validity of transcranial Doppler sonographic monitoring during cardiac surgery. Anesthesiology. 1994 Dec;81(6):1401-10.

10. Meng L, Hou W, Chui J, Han R, Gelb AW. Cardiac Output and Cerebral Blood Flow: The Integrated Regulation of Brain Perfusion in Adult Humans. Anesthesiology. 2015 Nov;123(5):1198-208.

11. Estrera AL, Garami Z, Miller CC III, Sheinbaum R, Huynh TTT, Porat EE, et al. Cerebral monitoring with transcranial Doppler ultrasonography improves neurologic outcome during repairs of acute type $A$ aortic dissection. J Thorac Cardiovasc Surg. 2005 Feb;129(2):277-85.

12. Denault AY, Shaaban-Ali M, Cournoyer A, Benkreira A, Mailhot T. Chapter 7 - Near-Infrared Spectroscopy. In: Prabhakar H, editor. Neuromonitoring Techniques [Internet]. Academic Press; 2018 [cited 2019 Aug 29]. p. 179-233. Available from: .

13. $10.1186 / \mathrm{s} 12871-019-0705-y$ 
Thudium M, Ellerkmann RK, Heinze I, Hilbert T. Relative cerebral hyperperfusion during cardiopulmonary bypass is associated with risk for postoperative delirium: a cross-sectional cohort study. BMC Anesthesiol [Internet]. 2019 Dec [cited 2019 Mar 10];19(1). Available from: .

14. Zanatta P, Messerotti Benvenuti S, Bosco E, Baldanzi F, Palomba D, Valfrè C. Multimodal brain monitoring reduces major neurologic complications in cardiac surgery. J Cardiothorac Vasc Anesth. 2011 Dec;25(6):1076-85.

\section{Figures}

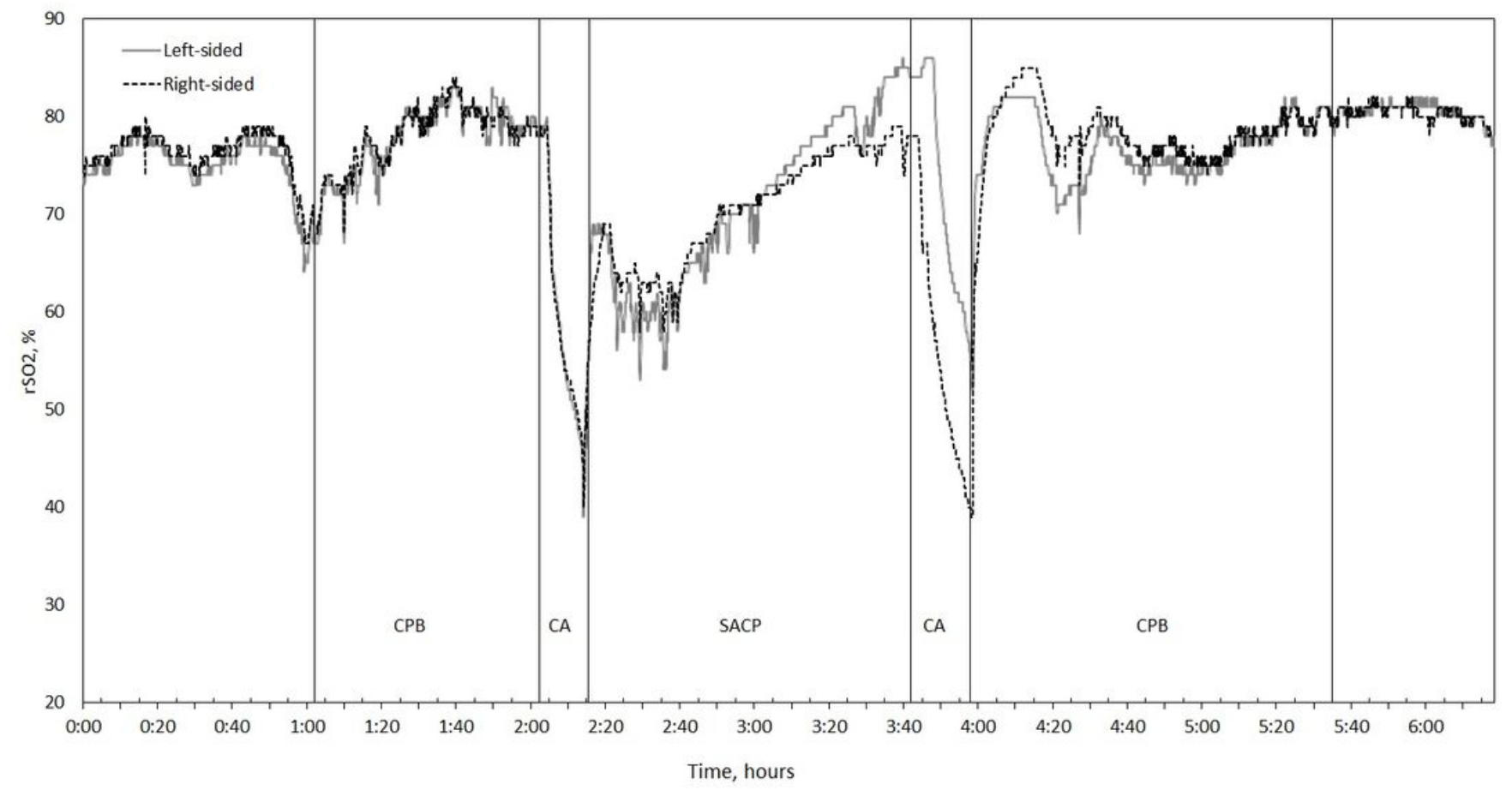

\section{Figure 1}

Representative Example of NIRS measurement during aortic arch repair procedure with corresponding operation phases (patient 1). CPB: cardiopulmonary bypass, CA: circulatory arrest, SACP: selective anterior cerebral perfusion 